\title{
Constrained field theories on Kerr backgrounds
}

\author{
Karan Fernandes $^{\mathrm{a}}$ (D) Amitabha Lahiri $^{\mathrm{b}}$ (D) \\ S N Bose National Centre for Basic Sciences, Block JD, Sector III, Salt Lake, Kolkata 700106, India
}

Received: 25 October 2018 / Accepted: 12 February 2019 / Published online: 21 February 2019

(c) The Author(s) 2019

\begin{abstract}
We analyze the constraints of gauge theories on Kerr and Kerr-de Sitter spacetimes, which contain one or more horizons. We find that the constraints are modified on such backgrounds through the presence of additional surface terms at the horizons. As a concrete example, we consider the Maxwell field and find that the Gauss law constraint involves surface corrections at the horizons. These surface contributions correspond to induced surface charges and currents on the horizons, which agree with those found within the membrane paradigm. The modification of the Gauss law constraint also influences the gauge fixing and Dirac brackets of the theory.
\end{abstract}

\section{Introduction}

The horizons of black holes are a profound consequence of the General Theory of Relativity. Black holes present to the universe a closed surface of finite size, completely characterized by macroscopic parameters such as mass, charge and spin [1]. Information about the internal structure of a black hole is unobservable from the outside due to the presence of the horizon, at least classically. The seminal discovery by Hawking [2] that a black hole radiates like a black body with a finite temperature, following Bekenstein's suggestion that a black hole possesses an entropy proportional to the surface area of its horizon [3], implies the possibility that a black hole has associated with it a very large number of microscopic states. It is natural to think that these states are in some way related to the degrees of freedom of the horizon. This view has been strengthened in approaches that treat fields on black hole backgrounds as those of manifolds with boundaries. For gravity, this approach leads to a quantum description in which an infinite set of observables are localized on the boundary [4-7].

\footnotetext{
${ }^{\mathrm{a}} \mathrm{e}-\mathrm{mail}$ : karan12t@bose.res.in

b e-mail: amitabha@boson.bose.res.in
}

There has been a resurgence of interest in studying the behaviour of quantum fields near black hole horizons, motivated by various paradoxes and puzzles related to the information problem [8,9]. Based on the asymptotic symmetries of fields on the null boundaries of conformally compactified flat spacetimes [10-15], there have been recent proposals for the existence of soft black hole hairs [16-20]. The significance of the horizon is highlighted in the membrane paradigm, where one replaces the black hole by a membrane with certain classical properties at the stretched horizon, i.e. a small distance outside the event horizon (an excellent overview is provided by the collection of articles in [21]). This is a sensible description from the perspective of an external stationary observer, who finds that particles cannot classically leave the interior of the black hole or reach the horizon from the outside in finite time. Thus the classical or semi-classical dynamics of fields on black hole backgrounds may be studied by considering the bulk and the horizon, and completely ignoring what happens in the interior of horizon.

Boundary conditions on the fields play a crucial role in all these investigations. In most of these papers, though not all of them, the fields (or their derivatives) are set to vanish on the horizon. For many field theories, this is a convenient way of ensuring that invariants constructed out of the stress energy tensor remain finite at the horizon. For the Kerr black hole spacetime, boundary conditions on the components of electric and magnetic fields relate the charge and surface currents at the horizon [22-24]. These conditions allow for the extraction of electromagnetic energy from Kerr black holes through a magnetic Penrose process [25]. The boundary conditions for gauge fields are special in that we can ensure the finiteness of gauge invariant observables without necessarily imposing the finiteness on the components of gauge fields. In addition, assuming any particular values for gauge fields is not particularly meaningful, as they are defined always up to gauge transformations.

Gauge theories are characterized by the presence of redundant degrees of freedom, which leads to the presence of con- 
straints. The formalism for studying the dynamics of constrained systems was discovered by Dirac [26] and independently by Bergmann et al. [27,28], and has been applied to numerous theories of interest over the years [29-31]. While the formalism for constrained field theories set up by Dirac generalizes to curved backgrounds [32], the more general formulation in terms of shift and lapse variables was introduced by Arnowitt et al. [33]. In particular, this formulation has been used to understand the initial value problem of fields theories [34], the behaviour of the fields near the horizons of stationary black hole spacetimes [35], and its quantization [36]. Until recently, a noticeable absence in the literature involved the formulation of constrained theories on curved backgrounds with horizons. The modification of constraints due to spatial boundaries on flat backgrounds were investigated in $[37,38]$, while in $[39,40]$ the quantization of the Chern-Simons theory on a disk and the role of boundaries on the vacuum structure of the theory has been covered in detail. It is the boundary conditions on gauge fields at the horizon that concerns us in this paper. The point is that the value of a gauge field at a boundary can be changed by a gauge transformation. The only way to fix the boundary value of a gauge field is to restrict to gauge transformations which vanish at the boundary. However, there is no sensible reason to do that when the said boundary is not a physical singularity, so it is sufficient to keep the gauge transformations regular at the horizon. We will find that this seemingly innocuous condition leads to a modification of the system of constraints when a horizon is present.

The formulation of gauge theories on spherically symmetric backgrounds with horizons was considered in [41], where it was found that the constraints received contributions from terms localized at the horizon. In particular, this is true for the Gauss law constraint in electrodynamics, which now has an additional contribution from the horizon. This resulted in a vanishing charge for an observer situated at the horizon of a Reissner-Nordström black hole, while not affecting the usual charge observed by the oberver at infinity. In the present work we investigate the classical constraints of electrodynamics in Kerr spacetimes. We will find that like in the static spherically symmetric case, the Gauss law constraint in the stationary axisymmetric spacetime picks up a horizon term. While it is an expected result, we think it was worthwhile to check that it was not an artifact of spherical symmetry. Thus gauge transformations which do not vanish on the horizon are allowed for axisymmetric black hole spacetimes. We will also show that the horizon term is equivalent to a 'surface' charge density on the horizon which is not visible to outside observers, and that it induces a 'surface' current density on the horizon.

The organization of our paper is as follows. In Sect. 2, we set up our notations and conventions for the analysis of constraints on the Kerr background. In Sect. 3, we consider Maxwell's theory and explicitly derive the surface contribu- tions to the constraint on the horizon. Gauge fixing is considered in both the radiation gauge and axial gauge. Finally in Sect. 5, we discuss the physical consequences of our results. This involves a modification of the usual solution for the electromagnetic scalar potential known in the absence of boundaries. The calculations behind certain results used in the main body of the paper appear in three Appendices.

\section{General algorithm}

\subsection{Kerr backgrounds}

Here we consider the description of hypersurfaces for the Kerr background which will be needed in our treatment of constrained field theories. The spacetime, which may possess one or more horizons (as in the Kerr-de Sitter case) admits two Killing vector fields: a stationary $\xi^{a}$ and an axial $\omega^{a}$, whose normalization we take to be

$$
\begin{aligned}
\xi_{a} \xi^{a} & =-\lambda^{2}, \\
\omega^{a} \omega_{a} & =f^{2} .
\end{aligned}
$$

The orbits of $\omega^{a}$ are taken to be closed, i.e. $\omega^{a}$ is periodic. The Killing vector fields mutually commute with each other,

$[\xi, \omega]^{a}=\xi^{b} \nabla_{b} \omega^{a}-\omega^{b} \nabla_{b} \xi^{a}=0$.

Kerr backgrounds admit spatial hypersurfaces which are tangent to $\omega^{a}$ and orthogonal to the vector (but not Killing) field

$\chi^{a}=\xi^{a}+\alpha \omega^{a}$,

where $\alpha$ is defined through the contraction of the Killing vectors

$\alpha=-\frac{1}{f^{2}} \xi_{a} \omega^{a}$.

We note that $\alpha$ in general is not a constant. It now follows that this vector is timelike in the region where $\lambda^{2}+\alpha^{2} f^{2}$ is positive, since

$\chi_{a} \chi^{a}=-\beta^{2}=-\left(\lambda^{2}+\alpha^{2} f^{2}\right)$

Despite being a combination of Killing vectors, $\chi^{a}$ itself is not Killing since

$£_{\chi} g_{c d}=2 \omega_{(c} \nabla_{d)} \alpha$.

$\chi^{a}$ only coincides with the Killing vector on those surfaces where $\beta^{2}=0$ and $\alpha$ is a constant, i.e. on the horizons of the spacetime [42]. Hence $\chi^{a}$ is timelike in the region outside the event horizon of asymptotically flat backgrounds, or in the 
general case of black hole de Sitter backgrounds, between the event horizon and the cosmological horizon.

It is straightforward to verify that $\chi^{a}$ satisfies the Frobenius condition

$\chi_{[a} \nabla_{b} \chi_{c]}=0$.

Thus $\chi^{a}$ is a timelike vector which is orthogonal to some spatial hypersurface $\Sigma$. Since $\chi^{a} \omega_{a}=0$, these hypersurfaces are also tangent to the Killing vector $\omega^{a}$. The projection operator on $\Sigma$ is given by

$h_{b}^{a}=\delta_{b}^{a}+\beta^{-2} \chi^{a} \chi_{b}$.

We assume that the spacetime is 'Kerr-like', i.e., an orthonormal basis on it is $\left\{\beta^{-1} \chi^{a}, f^{-1} \omega^{a}, \mu^{a}, v^{a}\right\}$, where the unit vectors $\left\{\mu^{a}, v^{a}\right\}$ are orthogonal to both $\xi^{a}$ and $\omega^{a}$ (and it follows, to $\chi^{a}$ ) and span an integral submanifold. Both the Kerr and Kerr-de Sitter spacetimes fall in this category. Using this basis, we can express the spatial projector given in Eq. (2.8) as

$h_{b}^{a}=f^{-2} \omega^{a} \omega_{b}+\mu^{a} \mu_{b}+v^{a} v_{b}$.

The Killing horizons $\mathcal{H}$ are closed, axially symmetric surfaces, which are submanifolds of $\Sigma$. The induced metric on $\mathcal{H}$ is given by

$\sigma_{a b}=h_{a b}-n_{a} n_{b}$,

where $n^{a}$ is the outward (inward) pointing unit spatial normal to the inner (outer) horizon of the background, satisfying $n_{a} n^{a}=1$. Since the horizon is axially symmetric and $\omega^{a}$ is tangent to the hypersurface $\Sigma$, it also follows that $n_{a} \omega^{a}=0$.

Using $h_{b}^{a}$ we can now project any spacetime tensor onto the spacelike hypersurface $\Sigma$. Denoting the covariant derivative on the hypersurface by $\mathcal{D}_{a}=h_{a}^{b} \nabla_{b}$, we have the following projection

$\mathcal{D}_{a} t_{b \ldots d}^{c \ldots e}=h_{a}^{a^{\prime}} h_{b}^{b^{\prime}} h_{c^{\prime}}^{c} \ldots h_{e^{\prime}}^{e} h_{d}^{d^{\prime}} \nabla_{a^{\prime}} T_{b^{\prime} \ldots d^{\prime}}^{c^{\prime} \ldots e^{\prime}}$,

where $T_{b \ldots d}^{c \ldots e}$ is a spacetime tensor and $t_{b . \ldots d}^{c \ldots e}$ denotes its projection on the spacelike hypersurface.

The time coordinate is measured along $\chi_{a}$ and is constant on the hypersurface $\Sigma$. In what follows we will consider the time evolution vector to be along $\xi^{a}$. With this choice, $\alpha \omega^{a}$ and $\beta$ represent what are known as the shift and lapse of the time evolution vector. It is the lapse function $\beta$ which vanishes at the horizons.

\subsection{Hamiltonian formulation}

We will now briefly review the Hamiltonian formalism for field theories on Kerr-like backgrounds. This will serve to familiarize ourselves with the concepts and notations needed to address constrained field theories. As mentioned above, time evolution is taken to be along $\xi^{a}$. This ensures that the fields evolve in time while the background on which they are defined remains fixed. More specific to the Hamiltonian formalism, it ensures that Hamilton's equations take their usual form without any modification of the (covariant) definition of the Poisson bracket. Thus for any field $\Phi_{A}$, we have

$\dot{\Phi}_{A}:=£_{\xi} \Phi_{A}=£_{\chi} \Phi_{A}-\mathfrak{L}_{\alpha \omega} \Phi_{A}$.

Here $£_{v}$ is the Lie derivative along the vector $v$, the index $A$ stands for a collection of all indices distinguishing the field, including internal and Lorentz indices, and $\Phi_{A}$ may be either bosonic or fermionic.

The action functional for a field $\Phi_{A}$ is given by the time integral of the Lagrangian $L$, or equivalently the integral of the Lagrangian density $\mathcal{L}$ over the four volume,

$S\left[\Phi_{A}\right]=\int d t L \equiv \int d t \int_{\Sigma} \beta d V_{x} \mathcal{L}\left(\Phi_{A}(x), \nabla_{a} \Phi_{A}(x)\right)$,

where $d V_{x}$ is the volume element on $\Sigma$ and $\mathcal{L}\left(\Phi_{A}(x)\right.$, $\left.\nabla_{a} \Phi_{A}(x)\right)$ is the Lagrangian density. Denoting the spacetime volume element in the orthonormal basis by $\epsilon_{a b c d}$ and the spatial volume element of the hypersurface by ${ }^{(3)} \epsilon_{b c d}$, we have

$\chi^{a} \epsilon_{a b c d}=\beta^{(3)} \epsilon_{b c d}=\xi^{a} \epsilon_{a b c d}$.

Thus the projected volume element has the correct form even though time evolution takes place along $\xi^{a}$ while it is $\chi^{a}$ which is orthogonal to $\Sigma$.

The canonically conjugate momenta $\Pi^{A}$ are defined as

$\Pi^{A}(\vec{x}, t)=\frac{\delta L}{\delta \dot{\Phi}_{A}(\vec{x}, t)}$,

where the functional derivative is an 'equal-time' derivative evaluated on the hypersurface $\Sigma$,

$\frac{\delta \Phi_{A}(\vec{x}, t)}{\delta \Phi_{B}(\vec{y}, t)}=\delta_{A}^{B} \delta(x, y)=\frac{\delta \dot{\Phi}_{A}(\vec{x}, t)}{\delta \dot{\Phi}_{B}(\vec{y}, t)}$.

The $\delta(x, y)$ in Eq. (2.16) is a three-dimensional covariant delta function on $\Sigma$, satisfying

$\int_{\Sigma} d V_{y} \delta(x, y) f(\vec{y}, t)=f(\vec{x}, t)$.

We will sometimes write $\vec{x}$ or even $(\vec{x}, t)$ as $x$, etc. as we have done above. 
The canonical Hamiltonian now follows from the Legendre transform

$H_{C}=\int_{\Sigma} d V_{x}\left(\Pi^{A} \dot{\Phi}_{A}\right)-L$

The Poisson bracket is defined on the hypersurface, which for two functionals $F\left(\Phi_{A}(x), \Pi^{A}(x)\right)$ and $G\left(\Phi_{A}(x), \Pi^{A}(x)\right)$ of the fields and their momenta is defined as

$[F, G]_{P}=\int d V_{z}\left[\frac{\delta F}{\delta \Phi_{A}(z)} \frac{\delta G}{\delta \Pi^{A}(z)}-\frac{\delta G}{\delta \Phi_{A}(z)} \frac{\delta F}{\delta \Pi^{A}(z)}\right]$.

This definition provides the canonical Poisson brackets between the fields and their momenta, which follows from setting $F=\Phi_{A}(\vec{x}, t)$ and $G=\Pi^{B}(\vec{y}, t)$

$\left[\Phi_{A}(\vec{x}, t), \Pi^{B}(\vec{y}, t)\right]_{P}=\delta_{A}^{B} \delta(x, y)$.

The Poisson bracket of any function or functional of the fields and momenta with the Hamiltonian provides its time evolution (this is justified in Appendix C)

$\dot{F}=\left[F, H_{C}\right]_{P}$.

This Hamiltonian provides a complete description of the dynamics of the system only if all the velocities are uniquely mapped into momenta through Eq. (2.15). This of course is not the case for constrained field theories. The constraints and dynamics of such theories can be determined from the Dirac-Bergmann formalism, which has been treated extensively in many excellent textbooks and reviews [29-31]. In this formalism, constraints are classified into two types, first class and second class. Second class constraints can always be eliminated by using Dirac brackets while first class constraints, apart from pathological counterexamples, generate gauge transformations. All constrained field theories of interest to us, at least in this paper, are thus gauge field theories. We will find that the constraints include non-vanishing contributions from the fields on the horizon of the spacetime, thereby modifying the familiar constraints of theories on backgrounds without boundaries.

In the following, we will demonstrate this by considering the Maxwell field.

\section{The Maxwell field}

The action for the Maxwell field is given by

$S_{E M}=\int d V_{4}\left(-\frac{1}{4} F_{a b} F_{c d} g^{a c} g^{b d}\right)$, where $d V_{4}$ is the four dimensional volume form on the manifold $\Sigma \times \mathbb{R}$, and $F_{a b}=2 \partial_{[a} A_{b]}$. From Eq. (2.12), we have

$$
\begin{aligned}
\dot{A}_{b} \equiv £_{\xi} A_{b} & =£_{\chi} A_{b}-\alpha £_{\omega} A_{b}-\left(A_{a} \omega^{a}\right) \nabla_{b} \alpha \\
& =\chi^{a} F_{a b}+\nabla_{b}\left(A_{a} \xi^{a}\right)-\alpha \omega^{a} F_{a b} .
\end{aligned}
$$

We now define the projected fields $e_{a}=-\beta^{-1} \chi^{c} F_{c a}, \phi=$ $A_{a} \xi^{a}$ and $f_{a b}=F_{c d} h_{a}^{c} h_{b}^{d}$. Using these definitions and the projection operator of Eq. (2.8) on the action in Eq. (3.1), we find

$$
\begin{aligned}
S_{E M}= & -\int d t \int_{\Sigma} d V_{x} \frac{\beta}{4} F_{a b} F^{c d}\left(h_{c}^{a} h_{d}^{b}-2 \beta^{-2} \chi^{a} \chi_{c} h_{d}^{b}\right. \\
& \left.+\beta^{-4} \chi^{a} \chi_{c} \chi^{b} \chi_{d}\right) \\
= & \int d t \int_{\Sigma} d V_{x} \beta\left[\frac{1}{2} e_{a} e^{a}-\frac{1}{4} f_{a b} f^{a b}\right] .
\end{aligned}
$$

The last term in the parenthesis in the first line of Eq. (3.3) does not contribute due to the antisymmetry of $F_{a b}$. We also have the following projection of Eq. (3.2)

$\dot{a}_{b}=-\beta e_{b}+\mathcal{D}_{b} \phi+\alpha f_{b a} \omega^{a}$.

Substituting Eq. (3.4) in Eq. (3.3), we can write the projected action as

$$
\begin{aligned}
S_{E M}= & -\int d t \int_{\Sigma} d V_{x} \frac{\beta}{4}\left[f_{a b} f^{a b}+2 \beta^{-2}\left(\dot{a}^{a}-\mathcal{D}^{a} \phi\right.\right. \\
& \left.\left.-\alpha f^{a b} \omega_{b}\right)\left(\dot{a}_{a}-\mathcal{D}_{a} \phi-\alpha f_{a c} \omega^{c}\right)\right] .
\end{aligned}
$$

We note that since $\dot{\phi}$ is absent in the projected action, its conjugate momentum is a constraint,

$\pi^{\phi}=\frac{\partial L_{E M}}{\partial \dot{\phi}}=0$

The momenta conjugate to the $a_{b}$ are given by

$\pi^{b}=\frac{\partial L_{E M}}{\partial \dot{a}_{b}}=-e^{b}$

The canonical Hamiltonian follows from the usual definition

$$
\begin{aligned}
H_{C}= & \int_{\Sigma} d V_{x}\left(\pi^{b} \dot{a}_{b}\right)-L \\
= & \int_{\Sigma} d V_{x}\left[\beta\left(\frac{1}{2} \pi^{b} \pi_{b}+\frac{1}{4} f_{a b} f^{a b}\right)\right. \\
& \left.+\pi^{b} \mathcal{D}_{b} \phi+\alpha \pi^{b} f_{b a} \omega^{a}\right] .
\end{aligned}
$$

The Hamiltonian comprises of the usual energy density along with an energy current $\alpha \pi^{b} f_{b a} \omega^{a}$ due to the non-vanishing 
shift vector of the background. This is a known current which has been found elsewhere in considerations of the Maxwell field on foliated backgrounds involving a non-vanishing shift vector $[35,43]$. By including the constraint of Eq. (3.6) to the canonical Hamiltonian, a new Hamiltonian is defined,

$$
\begin{aligned}
H_{0}= & \int_{\Sigma} d V_{x}\left[\beta\left(\frac{1}{2} \pi^{b} \pi_{b}+\frac{1}{4} f_{a b} f^{a b}\right)+\pi^{b} \mathcal{D}_{b} \phi\right. \\
& \left.+\alpha \pi^{b} f_{b a} \omega^{a}+v_{\phi} \pi^{\phi}\right],
\end{aligned}
$$

where $v_{\phi}$ is an undetermined multiplier. The canonical Poisson brackets of Eq. (2.20) are in this case

$$
\begin{aligned}
{\left[\phi(x), \pi^{\phi}(y)\right]_{P} } & =\delta(x, y) \\
{\left[a_{a}(x), \pi^{b}(y)\right]_{P} } & =\delta_{a}^{b} \delta(x, y) .
\end{aligned}
$$

\subsection{The Dirac-Bergmann formalism}

We will now determine all additional constraints of the theory and construct the unconstrained Hamiltonian through the Dirac-Bergmann formalism. This requires the Poisson brackets of the constraints with the Hamiltonian, which we will always evaluate with the help of smearing functions. As we will see, these smearing functions come from the same space as the functions of gauge transformations, i.e. the dual space of the space of the gauge generators [44]. Therefore we will not assume that these smearing functions vanish at the horizons, but only that they are regular there. We need to check that the constraints of the theory are obeyed at all times, or in other words, $\dot{\pi}^{\phi} \approx 0$. This is done using the Poisson bracket between $\pi^{\phi}$ and the Hamiltonian, and with the help of a smearing function $\epsilon$ as follows,

$$
\begin{aligned}
\int_{\Sigma} & d V_{y} \epsilon(y) \dot{\pi}^{\phi}(y) \\
& =\int_{\Sigma} d V_{y} \epsilon(y)\left[\pi^{\phi}(y), H_{0}\right]_{P} \\
& =\int_{\Sigma} d V_{y} \epsilon(y)\left[\pi^{\phi}(y), \int_{\Sigma} d V_{x} \pi^{b}(x) \mathcal{D}_{b}^{x} \phi(x)\right]_{P} \\
& =\oint_{\partial \Sigma} d a_{y} \epsilon(y) n_{b}^{y} \pi^{b}(y)+\int_{\Sigma} d V_{y} \epsilon(y)\left(\mathcal{D}_{b}^{y} \pi^{b}(y)\right) .
\end{aligned}
$$

In deriving the above result we used the canonical Poisson brackets given in Eq. (3.10) and an integration by parts. The $n_{b}$ involved in the surface integral over the horizons of the spacetime is the 'unit normal' to the surface of the horizon satisfying $n_{b} n^{b}=1$ and pointing into the region where $\chi^{a}$ is timelike. We are particularly interested in the case where $\partial \Sigma$ consists of an outer cosmological horizon and an inner black hole horizon. Here and for the rest of the paper, we have allowed the smearing functions and its derivatives to be non-vanishing but regular at the horizons. Then by the Schwarz inequality we have

$\left|n_{b} \pi^{b}\right| \leq \sqrt{\left|n_{b} n^{b}\right|\left|\pi_{b} \pi^{b}\right|}$

$n_{b} n^{b}=1$ and $\pi_{b} \pi^{b}=e_{b} e^{b}$ appears in the energy momentum tensor (more precisely in invariant scalars such as $T^{a b} T_{a b}$ ), and therefore may not diverge at the horizon. Thus when the smearing function $\epsilon$ is regular at the horizon, the surface integral provides a finite contribution from $\partial \Sigma$. Hence setting the right hand side of Eq. (3.11) to weakly vanish produces the constraint

$$
\begin{aligned}
\int_{\Sigma} d V_{x} \epsilon(x) \Omega_{2}(x)= & \int_{\Sigma} d V_{x} \epsilon(x) \mathcal{D}_{b}^{x} \pi^{b}(x) \\
& +\oint_{\partial \Sigma} d a_{x} \epsilon(x) n_{b}^{x} \pi^{b}(x) \approx 0,
\end{aligned}
$$

In the absence of a surface integral, as for spacetimes without boundaries, we would extract the smearing function $\epsilon(x)$ and write the constraint as a weakly vanishing distribution on $\Sigma$, with the understanding that manipulations involving the constraint requires a smearing function and integration over the volume. In the present case, we will express the constraint appearing in Eq. (3.13) as

$\Omega_{2}=\mathcal{D}_{b} \pi^{b}+\left.n_{b} \pi^{b}\right|_{\mathcal{H}} \approx 0$.

The notation $\left.\right|_{\mathcal{H}}$ symbolizes that this term must be integrated with respect to the area element at the horizons. In other words, while the usual Gauss' Law constraint holds for all points of $\Sigma$, the additional surface contribution in Eq. (3.14) must be considered for all points at the horizon $\partial \Sigma$. This is a key result of our paper.

It is now straightforward to verify that $\dot{\Omega}_{2}=\left[\Omega_{2}(x), H_{0}\right]_{P}$ $\approx 0$, which reveals that there are no further constraints. Thus the full Hamiltonian is given by

$$
\begin{aligned}
H_{T}= & \int_{\Sigma} d V_{x}\left[\beta\left(\frac{1}{4} f_{a b} f^{a b}+\frac{1}{2} \pi_{a} \pi^{a}\right)+v_{1}\left(\mathcal{D}_{b} \pi^{b}\right)\right. \\
& \left.+\pi^{b} \mathcal{D}_{b} \phi+\alpha \pi^{b} f_{b a} \omega^{a}+v_{\phi} \pi^{\phi}\right] \\
& +\int_{\partial \Sigma} v_{1} n_{b} \pi^{b} .
\end{aligned}
$$


The multipliers $v_{1}$ and $v_{\phi}$ may be determined by examining the equations of motion. The evolution of $\phi$ is given by

$$
\begin{aligned}
& \int_{\Sigma} d V_{y} \epsilon(y) \dot{\phi}(y) \\
& =\int_{\Sigma} d V_{y} \epsilon(y)\left[\phi(y), H_{T}\right]_{P} \\
& =\int_{\Sigma} d V_{y} \epsilon(y) \int_{\Sigma} d V_{x} v_{\phi}(x)\left[\phi(y), \pi^{\phi}(x)\right]_{P} \\
& =\int_{\Sigma} d V_{y} \epsilon(y) v_{\phi}(y),
\end{aligned}
$$

which tells us that we can set $\dot{\phi}=v_{\phi}$. The evolution of $a_{b}$ can also be determined in a similar manner,

$$
\begin{aligned}
\int_{\Sigma} & d V_{y} \epsilon(y) \dot{a}_{b}(y)=\int_{\Sigma} d V_{y}\left[\epsilon(y) a_{b}(y), H_{T}\right]_{P} \\
= & \int_{\Sigma} d V_{y} \epsilon(y)\left[\beta(y) \pi_{b}(y)+\mathcal{D}_{b}^{y} \phi(y)\right. \\
& \left.+\alpha f_{b a} \omega^{a}-\mathcal{D}_{b}^{y} v_{1}(y)\right] .
\end{aligned}
$$

Comparing this with Eq. (3.4), we deduce that $\mathcal{D}_{b} v_{1}=0$. With this choice, Eq. (3.17) produces

$\dot{a}_{b}=\beta \pi_{b}+\mathcal{D}_{b} \phi+\alpha f_{b a} \omega^{a}$,

and this can result by simply setting $v_{1}=0$. While this choice is not unique, we would always have $v_{1} \Omega_{2}=\pi^{b} \mathcal{D}_{b} v_{1}=0$. Hence the total Hamiltonian takes the form

$$
\begin{aligned}
H_{T}= & \int_{\Sigma} d V_{x}\left[\beta\left(\frac{1}{4} f_{a b} f^{a b}+\frac{1}{2} \pi_{a} \pi^{a}\right)+\pi^{b} \mathcal{D}_{b} \phi\right. \\
& \left.+\alpha \pi^{b} f_{b a} \omega^{a}+\dot{\phi} \pi^{\phi}\right] .
\end{aligned}
$$

The two first class constraints generate gauge transformations on the fields. By evaluating the Poisson bracket of the fields $\phi$ and $a_{b}$ with the general linear combination of the constraints $\rho=\epsilon_{1} \pi^{\phi}+\epsilon_{2} \Omega_{2}$, we find that

$$
\begin{aligned}
\delta \phi(y) & =[\phi(y), \rho(x)]_{P}=\epsilon_{1}(y) \\
\delta a_{b}(y) & =\left[a_{b}(y), \rho(x)\right]_{P}=-\mathcal{D}_{b}^{y} \epsilon_{2}(y) .
\end{aligned}
$$

The gauge transformations which leave the Lagrangian in Eq. (3.1) invariant are $\delta A_{b}=\nabla_{b} \epsilon$. By projecting this expression using Eq. (2.8) we have

$\delta\left(\phi+\alpha \omega^{a} a_{a}\right)=£_{\chi} \epsilon, \quad \delta a_{b}=\mathcal{D}_{b} \epsilon$.
Eq. (3.20) is equivalent to Eq. (3.21), provided we identify $\epsilon_{1}(y)=£_{\xi} \epsilon(y)$ and $\epsilon_{2}(y)=-\epsilon(y)$. Without the horizon term in the constraint, the gauge transformations will clearly not have the usual form unless $\epsilon$ is assumed to vanish on the horizon.

A remark regarding the Hamiltonian and its relation to time in this space is in order. Even though the integral defining the Hamiltonian in Eq. (3.19) is over $\Sigma$ which is orthogonal to $\chi$, and not to the timelike Killing vector field $\xi$, the time evolution generated by this Hamiltonian provides the correct form of the Maxwell equations. This is shown in Appendix C.

\subsection{Gauge fixing}

We will look at this theory in two different gauges - the radiation gauge and the axial gauge. In the radiation gauge the full set of constraints are $\Omega_{i} \approx 0$, with

$$
\begin{aligned}
& \Omega_{1}=\pi^{\phi} \\
& \Omega_{2}=\mathcal{D}_{a} \pi^{a}+\left.n_{a} \pi^{a}\right|_{\mathcal{H}} \\
& \Omega_{3}=\phi \\
& \Omega_{4}=\mathcal{D}^{b}\left(\beta a_{b}\right) .
\end{aligned}
$$

The first two are the gauge constraints of the theory already found in Eqs. (3.6) and (3.14), while $\Omega_{3}$ and $\Omega_{4}$ are the gaugefixing functions. We call this the radiation gauge because that is what it reduces to in flat space. The full set of constraints is second-class, requiring the construction of Dirac brackets.

The non-vanishing Poisson brackets of the constraints in Eq. (3.22) are

$$
\begin{aligned}
& {\left[\Omega_{1}(x), \Omega_{3}(y)\right]_{P}=-\delta(x, y),} \\
& {\left[\Omega_{2}(x), \Omega_{4}(y)\right]_{P}=\mathcal{D}_{a}\left(\beta \mathcal{D}^{a} \delta(x, y)\right) .}
\end{aligned}
$$

The first Poisson bracket is the canonical relation given in Eq. (3.10). The second Poisson bracket is calculated as follows.

$$
\begin{aligned}
& {\left[\int_{\Sigma} d V_{x} \eta(x) \Omega_{2}(x), \int_{\Sigma} d V_{y} \epsilon(y) \Omega_{4}(y)\right]_{P}} \\
& =\left[\int_{\Sigma} d V_{x} \eta(x) \mathcal{D}_{a}^{x} \pi^{a}(x)+\oint_{\partial \Sigma} \eta(x) n_{a}^{x} \pi^{a}(x),\right. \\
& \left.\int_{\Sigma} d V_{y} \epsilon(y) \mathcal{D}_{y}^{b}\left(\beta(y) a_{b}(y)\right)\right]_{P} \\
& =\left[\int_{\Sigma} d V_{x}\left(\mathcal{D}_{a}^{x} \eta(x)\right) \pi^{a}(x), \int_{\Sigma} d V_{y} \beta(y)\left(\mathcal{D}_{y}^{b} \epsilon(y)\right) a_{b}(y)\right]_{P}
\end{aligned}
$$




$$
\begin{aligned}
= & -\int_{\Sigma} d V_{y} \beta(y)\left(\mathcal{D}_{a}^{y} \eta(y)\right)\left(\mathcal{D}_{y}^{a} \epsilon(y)\right) \\
= & \oint_{\partial \Sigma} d a_{y} \epsilon(y) n_{y}^{a} \beta(y)\left(\mathcal{D}_{a}^{y} \eta(y)\right) \\
& +\int_{\Sigma} d V_{y} \epsilon(y) \mathcal{D}_{y}^{a}\left(\beta(y) \mathcal{D}_{a}^{y} \eta(y)\right) .
\end{aligned}
$$

By using Schwarz's inequality on the surface term in the last line above, we get

$$
\begin{aligned}
\left|n^{a} \beta D_{a}(\eta)\right|^{2} & \leq\left|n^{a} n_{a}\right| \beta^{2}\left|\left(D_{a} \eta\right)\left(D^{a} \eta\right)\right| \\
& =\beta^{2}\left(D_{a} \eta\right)\left(D^{a} \eta\right) .
\end{aligned}
$$

Due to the presence of $\beta^{2}$, the surface integral vanishes and only the second term of Eq. (3.24) contributes. The Poisson bracket in Eq. (3.24) can thus be written as

$$
\begin{aligned}
& {\left[\int_{\Sigma} d V_{x} \eta(x) \Omega_{2}(x), \int_{\Sigma} d V_{y} \epsilon(y) \Omega_{4}(y)\right]_{P}} \\
& =\int_{\Sigma} d V_{y} \epsilon(y) \int_{\Sigma} d V_{x} \eta(x)\left[\mathcal{D}_{y}^{a}\left(\beta(y) \mathcal{D}_{a}^{y} \delta(x, y)\right)\right],
\end{aligned}
$$

which corresponds to the result given in Eq. (3.23). The matrix of the Poisson brackets between these constraints have a non-vanishing determinant and is invertible. This matrix, $C_{\alpha \beta}(x, y)=\left[\Omega_{\alpha}(x), \Omega_{\beta}(y)\right]_{P}$, is given by

$$
C(x, y)=\left(\begin{array}{cccc}
0 & 0 & -\delta(x, y) & 0 \\
0 & 0 & 0 & \mathcal{D}_{a}\left(\beta \mathcal{D}^{a} \delta(x, y)\right) \\
\delta(x, y) & 0 & 0 & 0 \\
0 & -\mathcal{D}_{a}\left(\beta \mathcal{D}^{a} \delta(x, y)\right) & 0 & 0
\end{array}\right) .
$$

The dynamics of the gauge fixed theory is determined through Dirac brackets, whose definition requires the inverse of the matrix given in Eq. (3.27). The Dirac brackets of the theory for two dynamical entities $A$ and $B$ (which may be functions or functionals on phase space) is defined as

$$
\begin{aligned}
{[A, B]_{D}=} & {[A, B]_{P} } \\
& -\int_{\Sigma} d V_{u} \int_{\Sigma} d V_{v}\left[A, \Omega_{\alpha}(u)\right]_{P} C_{\alpha \beta}^{-1}(u, v) \\
& {\left[\Omega_{\beta}(v), B\right]_{P} . }
\end{aligned}
$$

Thus we need to find the inverse of the operator $\mathcal{D}_{a}\left(\beta \mathcal{D}^{a}\right)$. Let us formally write the inverse as $G(x, y)$, i.e.

$\mathcal{D}_{a}\left(\beta \mathcal{D}^{a} G(x, y)\right)=-\delta(x, y)$, for some scalar function $G(x, y)$. This is the timeindependent and axisymmetric Green's function for the spacetime Laplacian operator as can be easily verified by projecting it on the hypersurface. Thus the inverse of the matrix in Eq. (3.27), $C_{\alpha \beta}^{-1}(x, y)$, is now given by

$C^{-1}(x, y)=\left(\begin{array}{cccc}0 & 0 & \delta(x, y) & 0 \\ 0 & 0 & 0 & G(x, y) \\ -\delta(x, y) & 0 & 0 & 0 \\ 0 & -G(x, y) & 0 & 0\end{array}\right)$.

Using Eq. (3.30) in Eq. (3.28) we find that the non-vanishing Dirac brackets are

$\left[a_{a}(x), \pi^{b}(y)\right]_{D}=\delta(x, y) \delta_{a}^{b}-\mathcal{D}_{a}^{x}\left(\beta(y) \mathcal{D}_{y}^{b} G(x, y)\right)$.

The Green function involved in Eq. (3.31) has a known closed form expression outside the ergosphere on the Kerr background [45]. For the electromagnetic field on the Schwarzschild background, it is known that the Dirac bracket in the radiation gauge reduces to the Poisson bracket when either $\pi^{b}$ or $a_{a}$ is at the horizon [46]. This does not occur if we use a modified radiation gauge which involves a surface term at the horizon. Since expressions for the scalar Green function valid up to the horizon of a Kerr black hole are not known, such an analysis cannot be performed on axisymmetric spacetimes. We will however further elaborate on the implications of a radiation gauge with a surface term at the horizons in the discussion section of this paper.

Given that our background is axisymmetric, we will now further consider the axial gauge. Our consideration of the axial gauge will generalize the treatment provided in [30] about flat spacetime. We adopt the basis $\left\{\phi^{a}, \mu^{a}, v^{a}\right\}$ described in Sect. 2 and will consider Eq. (2.9) in the following equations. While it is possible to identify and select the axial direction by an appropriate choice of coordinates in the $\mu-v$ plane, we will keep the choice of direction open for the moment and give a prescription independent of that choice. Let us fix a 'generalized axial gauge' by setting to zero the component of $a_{a}$ along $\mu^{a}$. We then have the following set of constraints in this gauge [30]:

$$
\begin{aligned}
& \Omega_{1}=\pi^{\phi} \\
& \Omega_{2}=\mathcal{D}_{b} \pi^{b}+\left.n_{b} \pi^{b}\right|_{\mathcal{H}} \\
& \Omega_{3}=\mu^{a} a_{a} \\
& \Omega_{4}=\mu^{a} \mathcal{D}_{a} \phi+\beta \mu^{a} \pi_{a}+\alpha \mu^{a} f_{a c} \omega^{c} .
\end{aligned}
$$


The constraints have the non-vanishing Poisson brackets

$\left[\Omega_{1}(x), \Omega_{4}(y)\right]_{P}=-\mu^{a}(y) \mathcal{D}_{a}^{y} \delta(x, y)=\left[\Omega_{4}(x), \Omega_{1}(y)\right]_{P}$,

$\left[\Omega_{2}(x), \Omega_{3}(y)\right]_{P}=\mu^{a}(y) \mathcal{D}_{a}^{y} \delta(x, y)=\left[\Omega_{3}(x), \Omega_{2}(y)\right]_{P}$,

$\left[\Omega_{3}(x), \Omega_{4}(y)\right]_{P}=\beta(y) \delta(x, y)$.

The bracket $\left[\Omega_{2}(x), \Omega_{4}(y)\right]_{P}$ vanishes because we have assumed that there is no torsion,

$$
\begin{aligned}
{\left[\int_{\Sigma} d V_{x} \eta(x) \Omega_{2}(x), \int_{\Sigma} d V_{y} \epsilon(y) \Omega_{4}(y)\right]_{P} } \\
=\left[\int_{\Sigma} d V_{x} \eta(x) \mathcal{D}_{b}^{x} \pi^{b}(x)+\oint_{\partial \Sigma} d a_{x} \eta(x) n_{b}^{x} \pi^{b}(x),\right. \\
\\
\left.\int_{\Sigma} d V_{y} \epsilon(y)\left(\mu^{a} \mathcal{D}_{a} \phi+\beta \mu^{a} \pi_{a}+\alpha \mu^{a} f_{a c} \omega^{c}\right)(y)\right] \\
=\int_{\Sigma} d V_{x} \mathcal{D}_{a}^{x}(\eta(x)) \mathcal{D}_{b}^{x}\left(\alpha ( x ) \epsilon ( x ) \left(\mu^{b}(x) \omega^{a}(x)\right.\right. \\
\left.\left.\quad-\mu^{a}(x) \omega^{b}(x)\right)\right) \\
+\oint_{\partial \Sigma} d a_{x} \mathcal{D}_{a}^{x}(\eta(x)) n_{b}^{x}\left(\alpha(x) \epsilon(x)\left(\mu^{b}(x) \omega^{a}(x)\right)\right) \\
=-\int_{\Sigma} d V_{x} \alpha(x) \epsilon(x)\left(\mu^{b}(x) \omega^{a}(x)\right. \\
\left.\quad-\mu^{a}(x) \omega^{b}(x)\right) \mathcal{D}_{b}^{x} \mathcal{D}_{a}^{x}(\eta(x))=0 .
\end{aligned}
$$

The Poisson brackets of (3.33) lead to the following matrix, $\mu^{a}(y) \mathcal{D}_{a}^{y} q(x, y)=-\delta(x, y)$

$\mu^{a}(y) \mathcal{D}_{a}^{y} p(x, y)=-\beta(y) q(x, y)$

The expressions for $p$ and $q$ on the asymptotically flat Kerr background in Boyer-Lindquist coordinates are derived in Appendix B. Since Eq. (3.37) and Eq. (3.38) involve first order differential equations, their solutions will also exist on other Kerr-like backgrounds. Using the matrix of Eq. (3.36) and the constraints given in Eq. (3.32), we derive the following non-vanishing Dirac brackets for the fields,

$$
\begin{aligned}
& {\left[\phi(x), a_{b}(y)\right]_{D}} \\
& \quad=\mu_{b}(y) \beta(y) q(x, y)+\mathcal{D}_{b}^{y} p(x, y), \\
& {\left[\phi(x), \pi^{b}(y)\right]_{D}} \\
& \quad=\mathcal{D}_{a}^{y}\left(\alpha(y) q(x, y)\left(\mu^{a}(y) \omega^{b}(y)-\mu^{b}(y) \omega^{a}(y)\right)\right) \\
& \quad+\left.n_{a}^{y} \alpha(y) q(x, y)\left(\mu^{a}(y) \omega^{b}(y)\right)\right|_{\mathcal{H}}, \\
& {\left[a_{b}(x), \pi^{c}(y)\right]_{D}} \\
& \quad=\delta_{b}^{c} \delta(x, y)+\mu^{c}(y) \mathcal{D}_{b}^{y} q(x, y) .
\end{aligned}
$$

The Dirac bracket in Eq. (3.40), which also involves contributions from the horizons of the spacetime, is not present in flat space results involving the axial gauge. It appears here due to the non-vanishing shift vector of the Kerr background. The derivation of the bracket is provided in Appendix A.

Use of Dirac brackets ensures that all brackets involving $\mu^{a} a_{a}$, or the other constraints in Eq. (3.32), identically vanish. The Hamiltonian in Eq. (3.19) becomes, after the constraints of Eq. (3.32) have been imposed,

$C(x, y)=\left(\begin{array}{cccc}0 & 0 & 0 & -\mu^{a}(y) \mathcal{D}_{a}^{y} \delta(x, y) \\ 0 & 0 & \mu^{a}(y) \mathcal{D}_{a}^{y} \delta(x, y) & 0 \\ 0 & \mu^{a}(y) \mathcal{D}_{a}^{y} \delta(x, y) & 0 & \beta(y) \delta(x, y) \\ -\mu^{a}(y) \mathcal{D}_{a}^{y} \delta(x, y) & 0 & -\beta(y) \delta(x, y) & 0\end{array}\right)$.

The inverse of this matrix is needed for the Dirac brackets. Let us write it as

$C^{-1}(x, y)=\left(\begin{array}{cccc}0 & -p(x, y) & 0 & q(x, y) \\ p(x, y) & 0 & -q(x, y) & 0 \\ 0 & -q(x, y) & 0 & 0 \\ q(x, y) & 0 & 0 & 0\end{array}\right)$,

where $p(x, y)$ and $q(x, y)$ are two functions which may be found by evaluating $\int d V_{z} C(x, z) C^{-1}(z, y)=\delta(x, y)$. We find that these functions must satisfy

$$
\begin{aligned}
H_{T}= & \int_{\Sigma} d V_{x}\left[\beta\left(\frac{1}{4} f_{a b} f^{a b}+\frac{1}{2}\left(f^{-2} \omega^{a} \omega_{b}+v^{a} v_{b}\right) \pi_{a} \pi^{b}\right)\right. \\
& +\left(f^{-2} \omega^{a} \omega_{b}+v^{a} v_{b}\right) \pi^{b} \mathcal{D}_{a} \phi \\
& \left.-\frac{1}{2} \beta \mu^{a} \pi_{a} \mu^{b} \pi_{b}+\alpha v_{b} v^{c} \pi^{b} f_{c a} \omega^{a}\right] .
\end{aligned}
$$

\section{Charges and currents}

The modification of Gauss law by horizon terms has interesting consequences - in particular, it ties in nicely with the 
membrane paradigm as we shall see below. The electric and magnetic fields appearing in the analysis of constraints are in general from external sources, we do not assume that they share the symmetries of the background. We first note that Maxwell's equations resulting from the Hamiltonian, derived in Appendix C, are given by

$$
\begin{aligned}
& \mathfrak{£}_{\chi} \pi^{b}=\mathcal{D}_{a}\left(\beta f^{a b}\right)+\alpha \omega^{b}\left(\mathcal{D}_{a} \pi^{a}+\left.n_{a} \pi^{a}\right|_{\mathcal{H}}\right) \approx \mathcal{D}_{a}\left(\beta f^{a b}\right), \\
& £_{\chi} f_{a b}=2 \mathcal{D}_{[a} \beta \pi_{b]} .
\end{aligned}
$$

The equations of motion involve a term proportional to the Gauss law constraint, which does not affect the dynamics of $\pi^{b}$ since the constraint vanishes weakly. This is nothing unusual, Hamiltonian equations of motion hold up to constraints. Thus we find that for electromagnetism in black hole spacetimes, while the Gauss law constraint is modified by surface terms at the horizons, the dynamical Maxwell equations are not. The Gauss law constraint can be used to determine the charge contained in a given region of the spacetime. By considering a region from the black hole horizon $\mathcal{H}$ to an outer (spacelike) boundary $\partial \Sigma_{B}$, we have

$$
\begin{aligned}
Q_{B}= & \int_{\Sigma_{B}} d V_{x} \Omega_{2}(x) \\
= & \int_{\Sigma_{B}} d V_{x} \mathcal{D}_{b}^{x} \pi^{b}(x)+\oint_{\mathcal{H}} d a_{x} n_{b}^{x} \pi^{b}(x) \\
= & -\oint_{\partial \Sigma_{B}} d a_{x} n_{b}^{x} \pi^{b}(x)-\oint_{\mathcal{H}} d a_{x} n_{b}^{x} \pi^{b}(x) \\
& +\oint_{\mathcal{H}} d a_{x} n_{b}^{x} \pi^{b}(x)=-\oint_{\partial \Sigma_{B}} d a_{x} n_{b}^{x} \pi^{b}(x) .
\end{aligned}
$$

We have introduced the notation $\bar{\oint}$ to indicate any surface integral which arises from the horizon term in the Gauss law constraint. The surface integrals have their usual meaning and the notation is merely used to keep track of contributions from the surface terms in Gauss law. We see that for an observer outside the horizon, the enclosed charge is determined by the usual expression of the electric flux across $\partial \Sigma_{B}$. The surface term in the Gauss law constraint only contributes a surface integral at $\mathcal{H}$ and does not provide a term at $\partial \Sigma_{B}$. However, now let us shrink the surface to the horizon, $\partial \Sigma_{B} \rightarrow \mathcal{H}$. If we do the same calculation now, we will get an additional contribution $\overline{\oint_{\mathcal{H}}} d a_{x} n_{b}^{x} \pi^{b}(x)$ from the surface term in the Gauss law constraint, resulting in a vanishing charge at the black hole horizon
$Q_{\mathcal{H}}=0$

Thus the non-vanishing electric flux outside the horizon is seen to vanish by an observer at the horizon. Clearly it is the surface term in the constraint which causes the total charge to vanish for an observer on the horizon. This suggests that the surface term contribution in the Gauss law constraint corresponds to an induced charge on the horizon of the black hole. We can define the induced surface charge density $\sigma$ at the black hole horizon by

$\left.n_{b}^{x} \pi^{b}(x)\right|_{\mathcal{H}}=\left.\sigma(x)\right|_{\mathcal{H}}$

As the spacetime is rotating, we can also identify a surface current density on the horizon. Contracting Eq. (4.1) with the unit normal at the horizon, we find

$\mathfrak{£}_{\chi} \sigma=\mathcal{D}_{a}\left(\beta f^{a b} n_{b}\right)$

This equation represents the expression for charge conservation on Kerr spacetimes

$£_{\chi} \sigma+\mathcal{D}_{a} j^{a}=0$

provided we have

$\beta n_{a} f^{a b}=j^{b}$

as the induced current on the black hole horizon. Since the current is parallel to the horizon, $\mathcal{D}_{a} j^{a}$ is the twodimensional divergence on the surface of the horizon. If we define the magnetic field $B_{c}$ as

$\beta f^{a b}=-\epsilon^{a b c} B_{c}$,

then Eq. (4.8) is satisfied given the following expression for the parallel components of the magnetic field $B_{\|}^{a}$

$\left.B_{\|}^{a}\right|_{\mathcal{H}}=\left.\epsilon^{a b c} j_{b} n_{c}\right|_{\mathcal{H}}$

The above treatment extends to backgrounds with an outer cosmological horizon. In this case, by integrating the Gauss law over the entire hypersurface, whose inner boundary is the black hole horizon $\mathcal{H}_{1}$ and outer boundary is the cosmological horizon $\mathcal{H}_{2}$, we have

$$
\begin{aligned}
Q & =\int_{\Sigma} d V_{x} \Omega_{2}(x) \\
& =\int_{\Sigma} d V_{x} \mathcal{D}_{b}^{x} \pi^{b}(x)+\oint_{\mathcal{H}} d a_{x} n_{b}^{x} \pi^{b}(x)
\end{aligned}
$$




$$
\begin{aligned}
= & -\oint_{\mathcal{H}_{2}} d a_{x} n_{b}^{x} \pi^{b}(x)-\oint_{\mathcal{H}_{1}} d a_{x} n_{b}^{x} \pi^{b}(x)+\oint_{\mathcal{H}_{2}} d a_{x} n_{b}^{x} \pi^{b}(x) \\
& +\oint_{\mathcal{H}_{1}} d a_{x} n_{b}^{x} \pi^{b}(x)=0 .
\end{aligned}
$$

The surface charge density in Eq. (4.5) and surface current density in Eq. (4.10) can now be defined on both $\mathcal{H}_{1}$ and $\mathcal{H}_{2}$. We hence have the following situation. The Gauss law constraint on backgrounds with horizons is modified by surface terms at the horizons of the spacetime, which can be identified with induced surface charge densities defined locally on these horizons. These induced charges lead to a vanishing electric flux at the horizons and is related to the normal component of the electric field. On the other hand from Maxwell's equations, we can also determine induced surface current densities on the horizons of Kerr spacetimes. These current densities are related to parallel components of the magnetic field on the horizons.

The induced charges and currents that we find on black hole horizons have been introduced before in the literature. It was noted in [47] that when an electric charge is lowered into a Schwarzschild black hole, the electric flux lines terminate on the horizon. This required the introduction of an induced surface charge density on the horizon, and the electric potential was calculated as the superposition of that due to the external charge and that due to the induced charge. This result was generalized to describe an induced surface current density on the horizon of a rotating black holes in an asymptotically flat spacetime in [22,23]. The induced surface charges and currents can be described within the membrane paradigm as conditions on the electromagnetic fields on the membrane $[21,35]$ as well as through a surface action for the electromagnetic field on the membrane [48]. The induced charges and currents on the horizon help describe the Blandford-Znajek mechanism [25], a magnetic Penrose process which provides a model for the source of pulsars, quasars and active galactic nuclei $[49,50]$. Our result demonstrates that induced charges and currents on the horizon arise naturally as part of the general Gauss law constraint on black hole backgrounds. In the membrane paradigm, the induced charge density on the horizon appears as a consequence of boundary conditions . The vanishing electric flux at the horizons, following our treatment, could provide a means to investigate soft limits and their relation to gauge parameters at the horizon. In this regard, we note the proposal in [16], where soft hairs were defined as charges on the future horizon of the black hole, considered as a 'holographic plate', which are associated with non-vanishing large gauge transformations on the horizon. It will be interesting to investigate if such charges also result for the quantized electromagnetic field as a consequence of gauge parameters and constraints at the horizons.

\section{Discussion}

In this paper we have considered the constrained dynamics of field theories on Kerr backgrounds with one or more horizons and have argued that the constraints of the theory will receive additional contributions from these horizons. We explicitly considered the example of the Maxwell field, and found that the Gauss law constraint must include contributions from the horizon(s). Such surface contributions will not arise on spacelike surfaces of the background, but they appear on horizons in part due to our inability to observe past the horizon, as well as the fact that gauge fields can in principle take on arbitrary values at the horizon provided gauge invariant quantities constructed from them remain finite. More precisely, the non-vanishing of gauge parameters and their derivatives at the horizons leads to a Gauss law constraint with surface contributions.

A Gauss law constraint with horizon corrections implies some novel consequences on our understanding of the electromagnetic field, and more generally of gauge field theories, on black hole spacetimes. Let us briefly note the new results in this paper and how they relate with known results in the literature. Firstly, for gauge fields on spacetimes with horizons, existing literature sets gauge parameters or their derivatives to vanish on the horizon exactly as on spatial boundaries. Then the Gauss law constraint involves no surface contribution. A recent alternative approach introduces, at spatial boundaries, additional dynamical fields whose transformations cancel the surface contributions resulting from gauge transformations [51,52]. What we find, by not fixing the gauge parameters to vanish at a horizon, is that we can choose more general boundary conditions which allow not only horizon terms in the Gauss law constraint but also gauge fixing conditions which involve additional contributions from the horizon. As we discuss below, these terms could help explore the physical degrees of freedom at the Killing horizons.

A second novel result we have found is that the horizon contribution to the Gauss law leads to a vanishing electric flux across the horizon. Thus the horizon term can be interpreted as an induced 'surface' charge density at the horizon which in turn induces a surface current at the horizon. Previous work based on the membrane paradigm had postulated the existence of such a surface charge and surface current [23, 47]. We found that the same charge and current appears on the horizon as a consequence of the modification of Gauss law brought about by our choice to allow gauge parameters to not vanish on the horizon.

While some of the results in this paper are similar to those previously presented by some of us for spherically symmet- 
ric black hole spacetimes [41], the class of Kerr spacetimes considered in this paper lead to some interesting new results. The spatial hypersurfaces on which we have integrated the Hamiltonian density cannot be taken to be orthogonal to the timelike Killing vector field, but are orthogonal to a timelike vector field which coincides with the Killing field only at the horizons. Thus it is not a priori obvious that the unconstrained Hamiltonian would generate time evolution, but we have shown in Appendix $\mathrm{C}$ that this Hamiltonian does indeed produce Maxwell equations modulo first class constraints. Another key difference involves the implications of the Gauss law constraint on currents at Killing horizons. As we mentioned in the previous section, the surface terms in the Gauss law constraint can be directly associated with surface charges and currents postulated previously in the literature. A third difference is that there are many more gauge choices compatible with the symmetries of the Kerr-type background than for the spherically symmetric case. This also implies that surface terms in the gauge fixed theory are more general than those on spherically symmetric spacetimes, which we will now describe.

Some consequences of the modified Gauss law constraint can be determined by gauge fixing the theory. In Sect. (3) we considered two gauges - the radiation gauge and the axial gauge. For the radiation gauge considered in Eq. (3.22), we chose the covariant generalization of the gauge adopted in flat space. Unsurprisingly, the Dirac brackets for $a_{a}$ and $\pi^{b}$ in Eq. (3.31) are the covariant generalizations of the flat space result, involving the Green function of the spacetime Laplacian operator. As we noted in our treatment of this gauge however, it would be useful to include additional surface terms at the horizons. To see this, let us now consider the following gauge fixing function

$\Omega_{4}=\mathcal{D}^{b}\left(\beta^{-1} a_{b}\right)+\left.\beta^{-1} n^{b} a_{b}\right|_{\mathcal{H}}$,

in place of the expression in Eq. (3.22), with $\Omega_{3}$ as given. Unlike $\Omega_{4}$ in Eq. (3.22), this gauge function involves additional terms at the horizons. The time derivative of $\Omega_{4}$ gives

$\dot{\Omega}_{4}=\mathcal{D}_{a} \pi^{a}+\left.n^{a} \pi^{a}\right|_{\mathcal{H}}=\Omega_{2} \approx 0$

Thus this constraint is a consistent choice. Proceeding as before, we now find that the bracket of $\left[\Omega_{2}(x), \Omega_{4}(y)\right]$ is given by

$$
\begin{aligned}
{\left[\Omega_{2}(x), \Omega_{4}(y)\right]_{P}=} & \mathcal{D}_{a}^{x}\left(\beta^{-1}(x) \mathcal{D}_{x}^{a} \delta(x, y)\right) \\
& +\left.\beta^{-1}(x) n_{a}^{x} \mathcal{D}_{a}^{x} \delta(x, y)\right|_{\mathcal{H}} .
\end{aligned}
$$

From the Schwarz inequality, it follows that the surface term in Eq. (5.3) does not vanish. The resulting Dirac bracket will require the Green function for the operator involved in Eq. (5.3), which has a non-trivial surface contribution. This
Green function and its derivatives do not vanish at the horizons. Hence the horizons will affect the Dirac brackets and the dynamics of the theory.

A related point concerns the expression for the scalar potential $\phi$ following the axial gauge of Eq. (3.32)

$$
\begin{aligned}
\mathcal{D}_{b}\left(f^{-2} \omega^{b} \omega_{a} \pi^{a}+v^{b} v_{a} \pi^{a}\right)+\left.\left[n_{b} v^{b} v_{a} \pi^{a}\right]\right|_{\mathcal{H}} \\
=\mathcal{D}_{b}\left(\beta^{-1} \mu^{b} \mu^{a}\left(\mathcal{D}^{a} \phi+\alpha f_{a c} \omega^{c}\right)\right) \\
\quad+\left.\beta^{-1} n_{b} \mu^{b} \mu^{a}\left(\mathcal{D}_{a} \phi+\alpha f_{a c} \omega^{c}\right)\right|_{\mathcal{H}},
\end{aligned}
$$

where we made use of Eq. (2.9). From Eq. (5.4) it also follows that $\phi$ depends non-trivially on $\pi^{b}$ at the horizon. Thus in general, the horizon correction in the Gauss law constraint will manifest in the dependent variables of the theory following gauge fixing.

Another implication of the Gauss law constraint involves the charges and currents on Kerr spacetimes. We noted in Sect. 4 that the horizon correction in the Gauss law can be identified with the induced surface charge on the horizon of a black hole. This term was considered previously in the literature through boundary conditions on the normal component of the electric field. In addition, Maxwell's equations further imply an induced surface current as a consequence of the induced surface charge, which is related to components of the magnetic field parallel to the horizon. Thus corrections to the Gauss law constraint resulting from Killing horizons of the background lead to a natural identification of an induced surface charge and induced surface current in Eqs. (4.5) and (4.10) respectively. The induced surface charge in particular implies the vanishing of electric flux lines on the horizon. Non-vanishing gauge parameters are associated with soft charges at null infinity on asymptotically flat spacetimes [13]. It will be interesting to consider if the classically vanishing electric flux at Killing horizons, which arises as a consequence of non-vanishing gauge parameters following our treatment, is also related to a description of soft hairs on Killing horizons in the quantized theory [16].

Finally, we note that the BRST formalism provides an interesting and powerful means to investigate quantized fields in the Hamiltonian framework. Following our analysis in this paper and in [41], it can be argued that the BRST charge operator will involve the additional surface terms contained in the constraints. Thus the physical states defined by the cohomology of the BRST charge will have to satisfy non-trivial conditions on the horizon. Within the BRST formalism, we can expect that the surface corrections in the BRST charge and gauge-fixing fermion will affect the path integral and resulting quantum theory. The exploration of these issues lie outside the scope of the present work. We have recently considered some of these questions in the case of spherically symmetric spacetimes elsewhere [53]. We leave the investi- 
gation on axisymmetric spacetimes and physical states at the horizon for future work.

Data Availability Statement This manuscript has no associated data or the data will not be deposited. [Authors' comment: Data sharing not applicable to this article as no datasets were generated or analysed during the current study.]

Open Access This article is distributed under the terms of the Creative Commons Attribution 4.0 International License (http://creativecomm ons.org/licenses/by/4.0/), which permits unrestricted use, distribution, and reproduction in any medium, provided you give appropriate credit to the original author(s) and the source, provide a link to the Creative Commons license, and indicate if changes were made.

Funded by SCOAP ${ }^{3}$.

\section{Appendix A: Derivation of the Dirac bracket $\left[\phi(x), \pi^{b}(y)\right]_{D}$}

The second Dirac bracket provided in Eq. (3.41) is given by

$$
\begin{aligned}
& {\left[\phi(x), \pi^{b}(y)\right]_{D}} \\
& \quad=\mathcal{D}_{a}^{y}\left(\alpha(y) q(x, y)\left(\mu^{a}(y) \omega^{b}(y)-\mu^{b}(y) \omega^{a}(y)\right)\right) \\
& \quad+\left.n_{a}^{y} \alpha(y) q(x, y)\left(\mu^{a}(y) \omega^{b}(y)\right)\right|_{\mathcal{H}}
\end{aligned}
$$

Here we provide its derivation to elaborate on the surface term. From Eq. (3.28) we have

$$
\begin{aligned}
{\left[\phi(x), \pi^{b}(y)\right]_{D}=} & -\int_{\Sigma} d V_{u} \int_{\Sigma} d V_{v}\left[\phi(x), \Omega_{\alpha}(u)\right]_{P} \\
& C_{\alpha \beta}^{-1}(u, v)\left[\Omega_{\beta}(v), \pi^{b}(y)\right]_{P} .
\end{aligned}
$$

where we made use of the fact that $\left[\phi(x), \pi^{b}(y)\right]_{P}=0$. The expression in Eq. (A2) simplifies to

$$
\begin{gathered}
{\left[\phi(x), \pi^{b}(y)\right]_{D}=-\int_{\Sigma} d V_{u} \int_{\Sigma} d V_{v}\left[\phi(x), \Omega_{1}(u)\right]_{P}} \\
C_{14}^{-1}(u, v)\left[\Omega_{4}(v), \pi^{b}(y)\right]_{P} .
\end{gathered}
$$

Equations (3.32) and (3.36) can now be used to find the expression

$$
\begin{aligned}
& {\left[\phi(x), \pi^{b}(y)\right]_{D}} \\
& =-\int_{\Sigma} d V_{u} \delta(x, u) q(u, v) \int_{\Sigma} d V_{v} \alpha(v)\left(\mu^{a}(v) \omega^{b}(v)\right. \\
& \left.\quad-\mu^{b}(v) \omega^{a}(v)\right) \mathcal{D}_{a}^{v} \delta(v, y) \\
& =-\int_{\Sigma} d V_{v} q(x, v) \alpha(v)\left(\mu^{a}(v) \omega^{b}(v)\right. \\
& \left.\quad-\mu^{b}(v) \omega^{a}(v)\right) \mathcal{D}_{a}^{v} \delta(v, y) \\
& =\oint_{\partial \Sigma} d a_{v} \delta(v, y) n_{a}^{v} q(x, v) \alpha(v)\left(\mu^{a}(v) \omega^{b}(v)\right)
\end{aligned}
$$

$$
\begin{aligned}
& +\int_{\Sigma} d V_{v} \delta(v, y) \mathcal{D}_{a}^{v}\left(q ( x , v ) \alpha ( v ) \left(\mu^{a}(v) \omega^{b}(v)\right.\right. \\
& \left.\left.-\mu^{b}(v) \omega^{a}(v)\right)\right) .
\end{aligned}
$$

We made use of $n_{a} \omega^{a}=0$ in the last equality of Eq. (A4). Recalling that the brackets are in fact densities which need to be integrated over the hypersurface for both $x$ and $y$, we can express the result of Eq. (A4) as Eq. (A1).

\section{Appendix B: Axial gauge functions in Boyer-Lindquist coordinates}

We will now explicitly derive the functions $q(x, y)$ and $p(x, y)$ which appear in Eqs. (3.37) and (3.38) respectively. This will determine the inverse matrix given in Eq. (3.36) required to describe the Dirac brackets in the axial gauge. The Maxwell field is assumed to be defined on the Kerr background, for which we will adopt the usual Boyer-Lindquist coordinates $(t, r, \theta, \phi)$

$$
\begin{aligned}
d s_{B L}^{2}= & -\left(\frac{\Delta-a^{2} \sin ^{2} \theta}{\rho^{2}}\right) d t^{2} \\
& +\frac{2 a \sin ^{2} \theta}{\rho^{2}}\left(\Delta-r^{2}-a^{2}\right) d t d \phi \\
& +\frac{\sin ^{2} \theta}{\rho^{2}}\left(\left(r^{2}+a^{2}\right)^{2}-\Delta a^{2} \sin ^{2} \theta\right) d \phi^{2} \\
& +\frac{\rho^{2}}{\Delta} d r^{2}+\rho^{2} d \theta^{2},
\end{aligned}
$$

where

$$
\Delta=r^{2}-2 M r+a^{2}, \quad \rho^{2}=r^{2}+a^{2} \cos ^{2} \theta
$$

with $M$ being the mass of the black hole and $a$ the angular momentum per unit mass. In these coordinates the $r-\theta$ plane comprise the integral 2-submanifold orthogonal to both $t$ and $\phi$. Here we will explicitly derive the functions $q(x, y)$ and $p(x, y)$ corresponding to the axial gauge discussed in Sect. 3 , for the choices $\mu^{a}=\left(\partial_{\theta}\right)^{a}$ and $\nu^{a}=\left(\partial_{r}\right)^{a}$, i.e., for the gauge choice $a_{\theta}=0$. From the inverse metric in Boyer-Lindquist coordinates, we have

$$
\left(\partial_{r}\right)^{a}=\left(0, \frac{\sqrt{\Delta}}{\rho}, 0,0\right), \quad\left(\partial_{\theta}\right)^{a}=\left(0,0, \rho^{-1}, 0\right) .
$$

Likewise, the metric components of Eq. (B1) provide the following definitions 


$$
\begin{aligned}
\lambda^{2} & =-\frac{\Delta-a^{2} \sin ^{2} \theta}{\rho^{2}} \\
\alpha f^{2} & =-\frac{a \sin ^{2} \theta}{\rho^{2}}\left(\Delta-r^{2}-a^{2}\right) \\
f^{2} & =\frac{\sin ^{2} \theta}{\rho^{2}}\left(\left(r^{2}+a^{2}\right)^{2}-\Delta a^{2} \sin ^{2} \theta\right),
\end{aligned}
$$

as well as the following expressions for $\beta$ and $\sqrt{h}$

$$
\begin{aligned}
\beta & =\sqrt{-\left(\lambda^{2}+\alpha^{2} f^{2}\right)} \\
& =\left(1+\frac{4 M r\left(a^{2}+r^{2}\right)}{\Delta\left(a^{2} \cos (2 \theta)+a^{2}+2 r^{2}\right)}\right)^{-\frac{1}{2}} \\
\sqrt{h} & =\frac{f \rho^{2}}{\sqrt{\Delta}}
\end{aligned}
$$

Since Eq. (3.37) involves a delta function source, it will be convenient to first re-express it in terms of a second-order differential equation. Eq. (3.37) can be explicitly rewritten as

$$
\begin{aligned}
& \frac{1}{\rho\left(r^{\prime}, \theta^{\prime}\right)} \partial_{\theta^{\prime}}\left(\partial_{\theta^{\prime}} l\left(\vec{r}, \vec{r}^{\prime}\right)\right) \\
& =-\frac{1}{\sqrt{h\left(r^{\prime}, \theta^{\prime}\right)}} \delta\left(r-r^{\prime}\right) \delta\left(\theta-\theta^{\prime}\right) \delta\left(\phi-\phi^{\prime}\right),
\end{aligned}
$$

where we have chosen $q\left(\vec{r}, \vec{r}^{\prime}\right)=\partial_{\theta^{\prime}} l\left(\vec{r}, \vec{r}^{\prime}\right)$ and have considered the source at a fixed point $\vec{r}$. We now assume the following ansatz

$l\left(\vec{r}, \vec{r}^{\prime}\right)=l\left(r, \theta, \theta^{\prime}\right) \delta\left(r-r^{\prime}\right) \delta\left(\phi-\phi^{\prime}\right)$,

which simplifies Eq. (B6) to

$$
\begin{aligned}
& \delta\left(r-r^{\prime}\right) \frac{1}{\rho\left(r^{\prime}, \theta^{\prime}\right)} \partial_{\theta^{\prime}}\left(\partial_{\theta^{\prime}} l\left(r, \theta, \theta^{\prime}\right)\right) \\
& =-\frac{1}{\sqrt{h\left(r^{\prime}, \theta^{\prime}\right)}} \delta\left(r-r^{\prime}\right) \delta\left(\theta-\theta^{\prime}\right),
\end{aligned}
$$

The solution for $l\left(r, \theta, \theta^{\prime}\right)$ follows by first considering the homogeneous equation $\partial_{\theta^{\prime}}\left(\partial_{\theta^{\prime}} R\left(r, \theta, \theta^{\prime}\right)\right)=0$, whose general solution is

$$
R\left(r, \theta, \theta^{\prime}\right)=C_{1}(r, \theta)+C_{2}(r, \theta) \theta^{\prime}
$$

Requiring the solution for $l\left(r, \theta, \theta^{\prime}\right)$ to match across the point $\vec{r}=\vec{r}^{\prime}$, we can write the following general solution

$$
\begin{aligned}
l\left(r, \theta, \theta^{\prime}\right) & =C(r, \theta) \theta^{\prime} & & \left(\theta^{\prime}<\theta\right) \\
& =C(r, \theta) \theta & & \left(\theta^{\prime}>\theta\right),
\end{aligned}
$$

where $C(r, \theta)$ is a constant whose expression will be determined by substituting Eq. (B10) in Eq. (B8). Integrating $\phi^{\prime}$ and $r^{\prime}$ over their respective ranges, and $\theta^{\prime}$ from $\theta-\epsilon$ to $\theta+\epsilon$ (for an infinitessimal angle $\epsilon$ ), we find that the constant $C(r, \theta)$ is given by

$C(r, \theta)=\frac{\sqrt{\Delta(r, \theta)}}{f(r, \theta) \rho(r, \theta)}$,

where $f, \Delta$ and $\rho$ were defined in Eq. (B4) and Eq. (B2). With Eq. (B10), we now have the following general solution

$$
\begin{aligned}
l\left(\vec{r}, \vec{r}^{\prime}\right)= & \delta\left(r-r^{\prime}\right) \delta\left(\phi-\phi^{\prime}\right) \sqrt{\Delta(r, \theta)} \\
& (f(r, \theta) \rho(r, \theta))^{-1} \theta^{\prime} \quad\left(\theta^{\prime}<\theta\right) \\
= & \delta\left(r-r^{\prime}\right) \delta\left(\phi-\phi^{\prime}\right) \sqrt{\Delta(r, \theta)} \\
( & (f(r, \theta) \rho(r, \theta))^{-1} \theta \quad\left(\theta^{\prime}>\theta\right) .
\end{aligned}
$$

Differentiating this solution with respect to $\theta^{\prime}$ gives

$q\left(\vec{r}, \vec{r}^{\prime}\right)=\frac{\sqrt{\Delta(r, \theta)}}{f(r, \theta) \rho(r, \theta)} \Theta\left(\theta-\theta^{\prime}\right) \delta\left(r-r^{\prime}\right) \delta\left(\phi-\phi^{\prime}\right)$,

where $\Theta\left(\theta-\theta^{\prime}\right)$ is just the ordinary Heaviside step function. To derive the solution for $p(x, y)$, we differentiate Eq. (3.38) and use Eq. (3.37) to find

$\mu^{a}(y) \mathcal{D}_{a}^{y}\left(\beta^{-1}(y) \mu^{b}(y) \mathcal{D}_{b}^{y} p(x, y)\right)=\delta(x, y)$.

This equation can be solved using the procedure given above. In the case of $\mu^{a}=\left(\partial_{\theta}\right)^{a}$, Eq. (B14) becomes

$$
\begin{gathered}
\frac{1}{\rho\left(r^{\prime}, \theta^{\prime}\right)} \partial_{\theta^{\prime}}\left(\beta^{-1}\left(r^{\prime}, \theta^{\prime}\right) \frac{1}{\rho\left(r^{\prime}, \theta^{\prime}\right)} \partial_{\theta^{\prime}} p\left(\vec{r}, \vec{r}^{\prime}\right)\right) \\
=\frac{1}{\sqrt{h\left(r^{\prime}, \theta^{\prime}\right)}} \delta\left(r-r^{\prime}\right) \delta\left(\theta-\theta^{\prime}\right) \delta\left(\phi-\phi^{\prime}\right),
\end{gathered}
$$

whose solution is given by

$p\left(\vec{r}, \vec{r}^{\prime}\right)=\frac{\Delta(r, \theta)}{f(r, \theta) \rho(r, \theta)} \frac{F\left(\left|\theta-\theta^{\prime}\right| \mid \frac{a^{2} \Delta}{\left(a^{2}+r^{2}\right)^{2}}\right)}{\left(a^{2}+r^{2}\right)} \delta\left(r-r^{\prime}\right) \delta\left(\phi-\phi^{\prime}\right)$,

where $F\left(x \mid k^{2}\right)$ (with $k^{2}$ independent of $x$ ) is the elliptic integral of the first kind. If we consider Eq. (3.37) and Eq. (3.38) in flat spacetime, the solution for $q(x, y)$ involves the Heaviside step function and the solution of $p(x, y)$ involves the absolute value function, whose argument is the coordinate chosen in the axial gauge [30].The solutions for $q(x, y)$ in Eq. (B13) and $p(x, y)$ in Eq. (B16) are generalizations of these known solutions on the Kerr background. They can be substituted in Eq. (3.39), Eq. (3.40) and Eq. (3.41) to describe the Dirac brackets of the Maxwell field in the axial gauge. In the flat space limit $a, M \rightarrow 0$, we find $\Delta, \rho^{2} \rightarrow r^{2}, f \rightarrow r \sin \theta$, and it follows that $q(x, y)$ and 
$p(x, y)$ also agree with the corresponding flat space solutions.

\section{APPENDIX C: Maxwell equations}

Since the Hamiltonian $H$ involves an integral over the hypersurface $\Sigma$ which is orthogonal to $\chi$, it is not obvious that it does generate time evolutions along $\xi$, as indicated in 3. As a consistency check, we will here demonstrate that Maxwell's equations resulting from the Lagrangian also follow from the Hamiltonian if we set the time evolution generated by the Hamiltonian to be along $\xi$. By projecting $\nabla_{a} F^{a b}=0$ with Eq. (2.8), one can find the following projected Maxwell equations

$£_{\chi} e_{b}=-D_{a}\left(\beta f^{a b}\right)$

$£_{\chi} f_{a b}=-2 D_{[a} \beta e_{b]}$.

Turning our attention now to the Hamiltonian of Eq. (3.19), we find the following expressions upon evaluating the Poisson brackets

$$
\begin{aligned}
\dot{\pi}^{b}= & {\left[\pi^{b}, H_{T}\right]=\mathcal{D}_{a}\left(\beta f^{a b}\right)+\mathcal{D}_{a}\left(\alpha\left(\pi^{a} \omega^{b}-\pi^{b} \omega^{a}\right)\right) } \\
& +\left.\alpha n_{a} \pi^{a} \omega^{b}\right|_{\mathcal{H}}, \\
\dot{f}_{a b}= & {\left[f_{a b}, H_{T}\right]=2 \mathcal{D}_{[a} \beta \pi_{b]}+2 \mathcal{D}_{[a}\left(\alpha f_{b] c} \omega^{c}\right) . }
\end{aligned}
$$

It will be useful to note that since $\chi^{a} \omega_{a}=0, \omega^{c} \nabla_{c}=\omega^{c} \mathcal{D}_{c}$ on any function or tensor. Also from contracting Eq. (2.6), we see that $£_{\omega} \alpha=0$. Thus, $£_{\alpha \omega}$ of any spatially projected quantity can be written entirely in terms of the spatially projected covariant derivative. Let us first consider $\mathfrak{f}_{\alpha \omega} f_{a b}$

$$
\begin{aligned}
£_{\alpha \omega} f_{a b} & =\alpha \omega^{c} \mathcal{D}_{c} f_{a b}+f_{a c} \mathcal{D}_{b}\left(\alpha \omega^{c}\right)+f_{c b} \mathcal{D}_{a}\left(\alpha \omega^{c}\right) \\
& =2 \alpha \omega^{c} \mathcal{D}_{[b} f_{a] c}+f_{a c} \mathcal{D}_{b}\left(\alpha \omega^{c}\right)+f_{c b} \mathcal{D}_{a}\left(\alpha \omega^{c}\right) \\
& =-2 \mathcal{D}_{[a}\left(\alpha f_{b] c} \omega^{c}\right)
\end{aligned}
$$

where we made use of the Bianchi identity $\mathcal{D}_{[c} f_{a b]}=0$ in going from the first equality to the second equality of Eq. (C5). Likewise, we find for $£_{\alpha \omega} \pi^{b}$

$$
\begin{aligned}
£_{\alpha \omega} \pi^{b} & =\alpha \omega^{c} \mathcal{D}_{c} \pi^{b}-\pi^{c} \mathcal{D}_{c} \alpha \omega^{b} \\
& =\mathcal{D}_{c}\left(\alpha\left(\omega^{c} \pi^{b}-\pi^{c} \omega^{b}\right)\right)-\alpha \omega^{b} \mathcal{D}_{c} \pi^{c} .
\end{aligned}
$$

In going from the first equality to the final equation of Eq. (C6), we used the property that $\omega^{c}$ is Killing. Substituting Eq. (C5) in Eqs. (C4) and (C6) in Eq. (C3), we find

$$
£_{\chi} \pi^{b}=\mathcal{D}_{a}\left(\beta f^{a b}\right)+\alpha \omega^{b}\left(\mathcal{D}_{a} \pi^{a}+\left.n_{a} \pi^{a}\right|_{\mathcal{H}}\right) \approx \mathcal{D}_{a}\left(\beta f^{a b}\right),
$$

$£_{\chi} f_{a b}=2 \mathcal{D}_{[a} \beta \pi_{b]}$.

Substituting Eq. (3.7) in the above expressions, we get the projected Maxwell equations given in Eqs. (C1) and (C2). The derivation here should be contrasted with the analogous derivation on spherically symmetric backgrounds, where the time evolution vector is both Killing and orthogonal to the hypersurface. Thus, the foliation and time evolution as presented in this paper is consistent with the covariant Maxwell equations.

\section{References}

1. S. Chandrasekhar, The Mathematical Theory of Black Holes (Clarendon, Oxford, 1985)

2. S. W. Hawking, Particle Creation by Black Holes. Commun. Math. Phys. 43, 199 (1975). https://doi.org/10.1007/BF02345020 [Erratum: Commun. Math. Phys. 46, 206 (1976)]

3. J.D. Bekenstein, Black holes and entropy. Phys. Rev. D 7, 2333 (1973). https://doi.org/10.1103/PhysRevD.7.2333

4. A.P. Balachandran, L. Chandar, A. Momen, Edge states in gravity and black hole physics. Nucl. Phys. B 461, 581 (1996). https://doi. org/10.1016/0550-3213(95)00622-2

5. A. P. Balachandran, L. Chandar, A. Momen, Edge states in canonical gravity. Talk delivered at the Montreal-Rochester-SyracuseToronto (MRST) Conference, 1995. arXiv:gr-qc/9506006 (unpublished)

6. S. Carlip, Black hole entropy from conformal field theory in any dimension. Phys. Rev. Lett. 82, 2828 (1999). https://doi.org/10. 1103/PhysRevLett.82.2828

7. S. Carlip, Entropy from conformal field theory at Killing horizons. Class. Quant. Grav. 16, 3327 (1999). https://doi.org/10.1088/ 0264-9381/16/10/322

8. A. Almheiri, D. Marolf, J. Polchinski, J. Sully, Black holes: complementarity or firewalls? JHEP 1302, 062 (2013). https://doi.org/ 10.1007/JHEP02(2013)062

9. S.L. Braunstein, S. Pirandola, K. yczkowski, Better late than never: information retrieval from black holes. Phys. Rev. Lett. 110(10), 101301 (2013). https://doi.org/10.1103/PhysRevLett.110.101301

10. A.P. Balachandran, S. Vaidya, Spontaneous Lorentz violation in Gauge theories. Eur. Phys. J. Plus 128, 118 (2013). https://doi.org/ 10.1140/epjp/i2013-13118-9

11. M. Campiglia, A. Laddha, Asymptotic symmetries and subleading soft graviton theorem. Phys. Rev. D 90(12), 124028 (2014). https:// doi.org/10.1103/PhysRevD.90.124028

12. A. Strominger, Asymptotic symmetries of Yang-Mills theory. JHEP 1407, 151 (2014). https://doi.org/10.1007/ JHEP07(2014)151

13. T. He, P. Mitra, A.P. Porfyriadis, A. Strominger, New symmetries of massless QED. JHEP 1410, 112 (2014). https://doi.org/10.1007/ JHEP10(2014)112

14. A. Strominger, On BMS invariance of gravitational scattering. JHEP 1407, 152 (2014). https://doi.org/10.1007/ JHEP07(2014) 152

15. T. He, V. Lysov, P. Mitra, A. Strominger, BMS supertranslations and Weinbergs soft graviton theorem. JHEP 1505, 151 (2015). https:// doi.org/10.1007/JHEP05(2015)151

16. S.W. Hawking, M .J. Perry, A. Strominger, Soft hair on black holes. Phys. Rev. Lett. 116(23), 231301 (2016). https://doi.org/10.1103/ PhysRevLett.116.231301 
17. H. Afshar, S. Detournay, D. Grumiller, W. Merbis, A. Perez, D. Tempo, R. Troncoso, Soft Heisenberg hair on black holes in three dimensions. Phys. Rev. D 93(10), 101503 (2016). https://doi.org/ 10.1103/PhysRevD.93.101503

18. M. Mirbabayi, M. Porrati, Dressed hard states and black hole soft hair. Phys. Rev. Lett. 117(21), 211301 (2016). https://doi.org/10. 1103/PhysRevLett.117.211301

19. S.W. Hawking, M.J. Perry, A. Strominger, Superrotation charge and supertranslation hair on black holes. JHEP 1705, 161 (2017). https://doi.org/10.1007/JHEP05(2017)161

20. F. Tamburini, M. De Laurentis, I. Licata, B. Thid, Twisted soft photon hair implants on black holes. Entropy 19(9), 458 (2017). https://doi.org/10.3390/e19090458

21. K.S. Thorne, R.H. Price, D.A. Macdonald, Black Holes: the Membrane Paradigm (Yale University Press, New Haven, 1986), p. 367p

22. R.L. Znajek, The electric and magnetic conductivity of a Kerr hole. Mon. Not. R. Astron. Soc. 185(4), 833-840 (1978). https://doi.org/ $10.1093 / \mathrm{mnras} / 185.4 .833$

23. T. Damour, Black hole eddy currents. Phys. Rev. D 18, 3598 (1978). https://doi.org/10.1103/PhysRevD.18.3598

24. R.H. Price, K.S. Thorne, Membrane viewpoint on black holes: properties and evolution of the stretched horizon. Phys. Rev. D 33, 915 (1986). https://doi.org/10.1103/PhysRevD.33.915

25. R.D. Blandford, R.L. Znajek, Electromagnetic extractions of energy from Kerr black holes. Mon. Not. R. Astron. Soc. 179, 433 (1977)

26. P.A.M. Dirac, Generalized Hamiltonian dynamics. Can. J. Math. 2, 129 (1950). https://doi.org/10.4153/CJM-1950-012-1

27. P.G. Bergmann, Non-linear field theories. Phys. Rev. 75, 680 (1949). https://doi.org/10.1103/PhysRev.75.680

28. J.L. Anderson, P.G. Bergmann, Constraints in covariant field theories. Phys. Rev. 83, 1018 (1951). https://doi.org/10.1103/PhysRev. 83.1018

29. P.A.M. Dirac, Lectures on Quantum Mechanics (Yeshiva University, New York, 1964)

30. A.J. Hanson, T. Regge, C. Teitelboim, Constrained Hamiltonian Systems (Accademia Nazionale dei Lincei, Rome, 1976)

31. M. Henneaux, C. Teitelboim, Quantization of Gauge Systems (University Press, Princeton, 1992), p. 520

32. P.A.M. Dirac, The Hamiltonian form of field dynamics. Can. J. Math. 3, 1 (1951). https://doi.org/10.4153/CJM-1951-001-2

33. R.L. Arnowitt, S. Deser, C.W. Misner, The dynamics of general relativity. Gen. Relativ. Gravit. 40, 1997 (2008). https://doi.org/10. 1007/s10714-008-0661-1

34. J.A. Isenberg, J.M. Nester, Extension of the york field decomposition to general gravitationally coupled fields. Ann. Phys. 108, 368 (1977). https://doi.org/10.1016/0003-4916(77)90017-3

35. D. MacDonald, K.S. Thorne, Black-hole electrodynamics-an absolute-space/universal-time formulation. Mon. Not. R. Astron. Soc. 198, 345 (1982)

36. R. Sorkin, The quantum electromagnetic field in multiply connected space. J. Phys. A 12, 403 (1979). https://doi.org/10.1088/ 0305-4470/12/3/016

37. M.M. Sheikh-Jabbari, A. Shirzad, Boundary conditions as Dirac constraints. Eur. Phys. J. C 19, 383 (2001). https://doi.org/10.1007/ s100520100590
38. M. Zabzine, Hamiltonian systems with boundaries. JHEP 0010, 042 (2000). https://doi.org/10.1088/1126-6708/2000/10/042

39. A.P. Balachandran, L. Chandar, E. Ercolessi, T.R. Govindarajan, R. Shankar, Maxwell-Chern-Simons electrodynamics on a disk. Int. J. Mod. Phys. A 9, 3417 (1994). https://doi.org/10.1142/ S0217751X94001357

40. A.P. Balachandran, P. Teotonio-Sobrinho, The edge states of the BF system and the London equations. Int. J. Mod. Phys. A 8, 723 (1993). https://doi.org/10.1142/S0217751X9300028X

41. K. Fernandes, S. Ghosh, A. Lahiri, Constrained field theories on spherically symmetric spacetimes with horizons. Phys. Rev. D 95(4), 045012 (2017). https://doi.org/10.1103/PhysRevD.95. 045012

42. S. Bhattacharya, A. Lahiri, No hair theorems for stationary axisymmetric black holes. Phys. Rev. D 83, 124017 (2011). https://doi.org/ 10.1103/PhysRevD.83.124017

43. M. Bojowald, Canonical Gravity and Applications: Cosmology, Black Holes, and Quantum Gravity (Cambridge University Press, New York, 2010), p. 312

44. R. Benguria, P. Cordero, C. Teitelboim, Aspects of the Hamiltonian dynamics of interacting gravitational gauge and higgs fields with applications to spherical symmetry. Nucl. Phys. B 122, 61 (1977). https://doi.org/10.1016/0550-3213(77)90426-6

45. A.C. Ottewill, P. Taylor, Static Kerr Green's function in closed form and an analytic derivation of the self-force for a static scalar charge in Kerr space-time. Phys. Rev. D 86, 024036 (2012). https://doi. org/10.1103/PhysRevD.86.024036

46. K. Fernandes, A. Lahiri, The inverse spatial Laplacian of spherically symmetric spacetimes. Class. Quant. Grav. 34(17), 175004 (2017). https://doi.org/10.1088/1361-6382/aa7f61. [arXiv:1601.01442 [gr-qc]]

47. R.S. Hanni, R. Ruffini, Lines of force of a point charge near a Schwarzschild black hole. Phys. Rev. D 8, 3259 (1973). https:// doi.org/10.1103/PhysRevD.8.3259

48. M. Parikh, F. Wilczek, An action for black hole membranes. Phys. Rev. D 58, 064011 (1998). https://doi.org/10.1103/PhysRevD.58. 064011. arXiv:gr-qc/9712077

49. J. Granot, T. Piran, O. Bromberg, J .L. Racusin, F. Daigne, Gamma-ray bursts as sources of strong magnetic fields. Space Sci. Rev. 191(1-4), 471 (2015). https://doi.org/10.1007/ s11214-015-0191-6. arXiv:1507.08671 [astro-ph.HE]

50. T. Piran, Magnetic fields in gamma-ray bursts: a Short overview. AIP Conf. Proc. 784, 164 (2005). https://doi.org/10.1063/1. 2077181. arXiv:astro-ph/0503060

51. W. Donnelly, L. Freidel, Local subsystems in gauge theory and gravity. JHEP 1609, 102 (2016). https://doi.org/10.1007/ JHEP09(2016)102. arXiv:1601.04744 [hep-th]

52. M. Geiller, Edge modes and corner ambiguities in $3 \mathrm{~d}$ ChernSimons theory and gravity. Nucl. Phys. B 924, 312 (2017). https://doi.org/ 10.1016/j.nuclphysb.2017.09.010. arXiv:1703.04748 [gr-qc]

53. K. Fernandes, A. Lahiri, Hamiltonian BRST formalism for gauge fields on black hole spacetimes. S.N.Bose Centre. arXiv:1809.05729 [gr-qc] (preprint) 